\title{
Magnetic fields of rapidly oscillating Ap stars
}

\author{
G. Mathys \\ European Southern Observatory, Chile
}

Magnetic field appears to play a major rôle in the pulsations of rapidly oscillating Ap (roAp) stars. Understanding of the behaviour of these objects thus requires knowledge of their magnetic field. Such knowledge is in particular essential to interpret the modulation of the amplitude of the photometric variations (with a frequency very close to the rotation frequency of the star) and to understand the driving mechanism of the pulsation. Therefore, a systematic programme of study of the magnetic field of roAp stars has been started, of which preliminary (and still very partial) results are presented here.

Magnetic fields of Ap stars can be diagnosed from the Zeeman effect that they induced in spectral lines either from the observation of line-splitting in high-resolution unpolarized spectra (which only occurs in favourable circumstances) or from the observation of circular polarization of the lines in medium- to high-resolution spectra.

The best studied roAp star, both from the point of view of the oscillations as from that of the magnetic field, is HD 83368. From circular polarization observations, the magnetic field of this star does not seem to have cylindrical symmetry about an axis passing through the centre of the star. Additional observations are being obtained to establish this more definitely and allow detailed modelling of the field. It can already be noticed, however, that this lack of symmetry, if confirmed, might possibly account for the presence of spherical harmonics with even values of $\ell$ in the deconvolution of the observed pulsations (Kurtz 1992).

In high-resolution $\left(\lambda / \Delta \lambda=10^{5}\right)$ unpolarized spectra, HD 134214, HD 137949, and HD 201601 are observed to have lines resolved into several magnetically split components, while HD 24712 and HD 176232 are found to have sharp unresolved lines. The lines of HD 128898 are obviously significantly broadened by rotational Doppler effect. This is puzzling as the variations of the longitudinal field of this star (measured from circularly polarized spectra), presumably due to its rotation, seem to be inconsistently slow. HD 24712 and HD 201601 have also been observed in circular polarization. In the former, the maximum of the mean longitudinal magnetic field occurs at the same phase as the pulsation amplitude maximum. The latter appears to have a rotation period of the order of a century.

More details about these magnetic observations are to be found in Mathys (1991; 1992, to be submitted to Astron. Astrophys.), and Mathys et al. (1992).

\section{References:}

Kurtz, D., 1992, Monthly Not. r. Astron. Soc., in press.

Mathys, G., 1991, Astron. Astrophys. Suppl., 89, 121.

Mathys, G., Landstreet, J.D., Lanz, T., 1992, IAU Coll. 138, in press. 